\title{
A.B. KOMAPOB,
}

к.т.Н., заместитель руководителя отдела ФГБНУ «Дирекция НТП» Минобрнауки России, г. Москва, Россия, abkom@fentp.ru

\section{A.H. ПETPOB,}

к.х.Н., генеральный директор ФГБНУ «Дирекция НТП» Минобрнауки России, г. Москва,

Россия, petrov@fentp.ru

\section{A.B. СAPTOPИ,}

к.ф.-М.Н., специалист отдела информационно-аналитического и организационного

обеспечения ФГБНУ «Дирекция НТП» Минобрнауки России, г. Москва, Россия,

sartoriandrey@gmail.com

\section{МОДЕЛЬ КОМПЛЕКСНОЙ ОЦЕНКИ ТЕХНОЛОГИЧЕСКОЙ ГОТОВНОСТИ ИННОВАЦИОННЫХ НАУЧНО- ТЕХНОЛОГИЧЕСКИХ ПРОЕКТОВ*}

\footnotetext{
УДК 338.28, 378.4

Комаров А.В., Петров А.Н., Сартори А.В. Модель комплексной оценки технологической готовности инновационных научно-технологических проектов (ФГБНУ «Дирекция НТП» Минобрнауки России, ул. Пресненский Вал, д. 19, стр. 1, г. Москва, Россия, 123557)

Аннотация. В статье описана модель комплексной оценки технологической готовности инновационных научнотехнологических проектов, являющаяся составной частью методики экспертной оценки проектов. Модель может I быть использована как на этапе отбора заявок на финансирование проектов, так и для создания инструментов I измерения уровня технологической готовности проектов в ходе их выполнения. Применение предложенной I модели позволит сформировать единые унифицированные подходы и требования к формированию системы I критериев для экспертной оценки инновационных научно-технологических проектов на различных стадиях их I жизненного цикла, которые могут быть использованы различными институтами развития, а для исполнителей 1 проектов - определить степень готовности полученных результатов к промышленному внедрению.

I Ключевые слова: научно-технологический проект, уровень готовности технологии, индекс технологической I готовности, TPRL, TRL, УГT, инновация, анализ, модель.

DOI 10.22394/2410-132X-2017-4-1-47-57

Цитирование публикации: Комаров А.В., Петров А.Н., Сартори А.В. (2018) Модель комплексной оценки технологиI ческой готовности инновационных научно-технологических проектов // Экономика науки. Т. 4. № 1. С. 47-57.
}

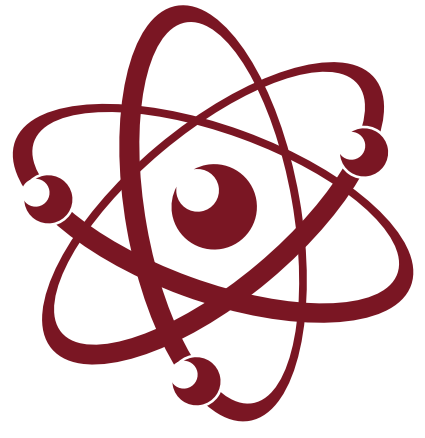

(C) А.В. Комаров, А.Н. Петров, А.В. Сартори, 2018 г.

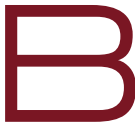

настоящее время, как в России, так и за рубежом, для оценки уровня готовности технологии используется хорошо зарекомендовавший себя подход, основанный на применении 9-ти уровневой шкалы TRL (Technology Readiness Level) [1] или ее российского аналога УГТ (Уровень Готовности Технологии), позволяющей различным группам специалистов (руководителям, менеджерам, администраторам, исследователям, конструкторам, технологам и т.д.) понимать, насколько данная технология в своем развитии продвинулась от научной идеи до практического применения. Впервые данная шкала была представлена в 80-х гг. прошлого столетия Национальным аэрокосмическим агентством США NASA [2], а ее использование оказалось успешным благодаря таким ее преимуществам, как:

\footnotetext{
Публикация выполнена при финансовой поддержке Минобрнауки России за счёт средств субсидии на выполнение государственного задания № 074-00522-18-02.
} 
- сравнение технологий - т.е. способности определять: готова ли одна технология к рынку так же, как и другая;

- последовательное улучшение результатов проекта, в т.ч. определение необходимых шагов для этого;

- проведение самооценки проекта - насколько проект соответствует портфелю проектов;

- упрощение коммуникаций - различные специалисты могут использовать единую метрику;

- $\quad$ адаптация шкалы под нужды конкретных отраслей или организаций с учетом их специфических требований.

В табл. 1 приведены сведения о характеристиках уровней TRL, используемых NASA в современной практике [3].

Следует отметить, что использование данной шкалы в различных государствах или крупных компаниях в настоящее время регулируется различными национальными или отраслевыми (корпоративными) стандартами [4], в России это такие стандарты, как ГОСТ Р 56861-2016 [5] и ГОСТ Р 57194.1-2016 [6].

Анализируя данные табл. 1 нетрудно заметить, что шкала TRL не охватывает достаточно много аспектов, которые следовало бы учитывать при оценке проекта в целом, в рамках которого разрабатывается инновационная технология, поэтому на практике при оценке проекта в целом используются подходы, основанные на шкале TRL, но описывающие также и другие уровни готовности, такие, например, как системная готовность (SRL), интеграционная готовность (IRL), производственная готовность (MRL) и ряд других, применяемых одновременно с TRL [7]. Так, например, для оценки проектов федеральной целевой программы «Исследования и разработки по приоритетным направлениям научно-технологического развития России на 2014-2020 годы» (далее ФЦП ИР) на основе метода TRL была разработана методология TPRL (Technology Project Readiness Level) [8], учитывающая такие ценности проекта, как:

- Технологическая готовность (TRL);

- Производственная готовность (MRL);

- Инженерная готовность (ERL);

- Организационная готовность (ORL);

- Преимущества и риски (BRL);

- Рыночная готовность и коммерциализация (CRL).

Преимуществом данной методологии является то обстоятельство, что при оценке уровень готовности проектов используются не только критерии, характеризующие тот или иной уровень готовности (как это принято в методе TRL), а и документы, на основании которых подтверждается выполнение данных критериев, причем эта оценка делается независимыми экспертами. Другой особенностью методологии TPRL является то, что она практически не применима на этапе отбора проектов для поддержки, в т.ч. финансовой, так как требования, предъявляемые к описанию проектов, не гармонизированы с содержанием критериев и показателей методологии TPRL, а даже наличие формализованных документов не предполагает оценку их качества, как это сделано, например, в [9], [10]. Кроме того, описания критериев и показателей,

Таблица 1

\section{Характеристика уровней TRL}

\begin{tabular}{c|l}
\hline TRL & \multicolumn{1}{c}{ Definition } \\
\hline 1 & Basic principles observed and reported \\
\hline 2 & Technology concept and/or application formulated \\
\hline 3 & Analytical and experimental critical function and/or characteristic proof-of-concept \\
\hline 4 & Component and/or breadboard validation in laboratory environment \\
\hline 5 & Component and/or breadboard validation in relevant environment \\
\hline 6 & System/subsystem model or prototype demonstration in a relevant environment (Ground or Space) \\
\hline 7 & System prototype demonstration in a space environment \\
\hline 8 & Actual system completed and "flight qualified" through test and demonstration (Ground or Flight)
\end{tabular}


характеризующих различные уровни готовности TPRL, носят достаточно общий характер, что на практике может приводить к тому, что оценка уровня готовности может быть сделана с высокой погрешностью.

Отметим также, что большинство известных на сегодняшний день информационно-технологических инструментов для оценки TRL (например, такие, как [11], [12], [13]), используемых для решения узкоотраслевых задач, построены на основе модификации т.н. калькулятора AFRL [14], созданного в среде табличного редактора MS Excel.

В связи с этим, разработка модели, позволяющей проводить комплексную оценку технологической готовности научно-технологических проектов, является актуальной, т.к. она может быть основой унифицированного информационно-технологического инструмента для оценки TRL, который может применяться в различных институтах развития для проектов в различных технических дисциплинах и разделах науки.

\section{1.Требования к разработке моде- ли комплексной оценки технологиче- ской готовности инновационных науч- но-технологических проектов}

Анализ опыта апробации методологии TPRL, проведенной в рамках программы акселерации проектов ФЦП ИР [15], а также применение ее упрощенного варианта при проведении совместного исследования SAP и НП «Руссофт», посвященного анализу перспектив российских ИТ-разработок на глобальном рынке [16], позволил определить требования к разработке модели комплексной оценки технологической готовности инновационных научно-технологических проектов. Модель должна:

- основываться на 9-ти уровней шкале оценки TRL, не исключая при этом возможности адаптации ее содержания под требования конкретной программы поддержки, реализуемой различными институтами развития;

- иметь универсальную структуру - т.е. она должна быть построена таким образом, что ее можно было бы применять для оценки технологической готовности проектов специалистами различных институтов развития и организаций и ее структура не должна быть связана с определенной технической дисциплиной и конкретным разделом науки;

- максимально полно и точно учитывать современные технологии и стандарты разработки с учетом жизненного цикла инновационного проекта (ГОСТ Р серии 1, ГОСТ серии 2, ГОСТ серии 3, ГОСТ 4, ГОСТ 14, ГОСТ 15, ГОСТ 17, ГОСТ 19, ГОСТ 20, ГОСТ 24, ГОСТ 27, ГОСТ 28, ГОСТ 29, ГОСТ 40, ГОСТ 50, и т.п.) - это позволит, с одной стороны, формализовать требования к составу и структуре документов, подтверждающих достижение того или иного уровня технологической готовности, а с другой - разработать систему подтверждения определения уровня технологической готовности;

- реализовывать механизмы как самостоятельной оценки, проводимой разработчиками (далее самооценки), так и экспертной оценки, осуществляемой независимой третьей стороной на базе единой инфологической модели, - т.е. в ней используются единая система критериев и показателей для оценки достижения определенного уровня технологической готовности как для разработчиков, так и для экспертов;

- включать критерии и показатели для оценки уровня технологической готовности, содержание которых должно быть максимально формализовано, что позволит исключить неоднозначное понимание характеристик различных уровней технологической готовности;

- учитывать наряду с характеристиками, присущими непосредственно TRL, и другие аспекты комплексной оценки проекта - т.е. в ней должны проводиться оценки и других свойств проекта, входящих в шкалу TPRL. Отметим, что набор таких характеристик должен быть достаточно универсальным, чтобы его можно было применять для оценки проектов различными институтами развития, не исключая при этом возможности его адаптации под их специфические требования;

- иметь вероятностную оценку расчета численных показателей, основанную на экспертной оценке качества подтверждающей информации о достижении того или иного результата для последующей оценки уровня технологической готовности; 
- иметь систему подтверждения правильности достижения определенных результатов, основанную на применении маркеров, учитывающих последовательность достижения определенных характеристик какого-либо уровня технологической готовности и указывающих на возможные преувеличения;

- предоставлять инструменты для мониторинга результативности и хода выполнения проектов в рамках текущего уровня технологической готовности проекта на малых временных интервалах или же рейтнингования проектов при принятии решений о предоставлении им поддержки.

\section{2. Структура модели}

С точки зрения особенностей анализа и обобщения информации при определении достижимости определенного уровня технологической готовности проекта, целесообразно представить модель комплексной оценки технологической готовности инновационных научно-технологических проектов в виде иерархической структуры, представленной на рис. 1.
Высший уровень иерархии (У1) содержит дискретный уровень технологической готовности проекта (TRL), значение которого изменяется от 1 до 9. Если в отношении проекта не подтвержден ни один из показателей, характеризующий уровень TRL равный 1, то значение уровня TRL принимается равным 0. Это позволяет помимо уровня технологической готовности проекта определять дробный индекс технологической готовности проекта, целая часть которого равна достигнутому уровню технологической готовности (или 0), а дробная часть характеризует динамику выполнения проекта на малых временных интервалах. Очевидно, что индекс технологической готовности проекта может быть использован как для мониторинга хода выполнения работ в рамках проекта, так и для рейтингования проектов.

$\mathrm{Ha}$ следующем уровне иерархии (У2) определяются показатели TRL и маркеры, позволяющие определить правильность определения показателей TRL. Показатели TRL определяют этапы развития проекта в пределах одного уровня технологической готовности, например, «Проведен анализ

\section{Экспертная оценка Самооценка}

Элементы модели (использование элементов (использование элементов

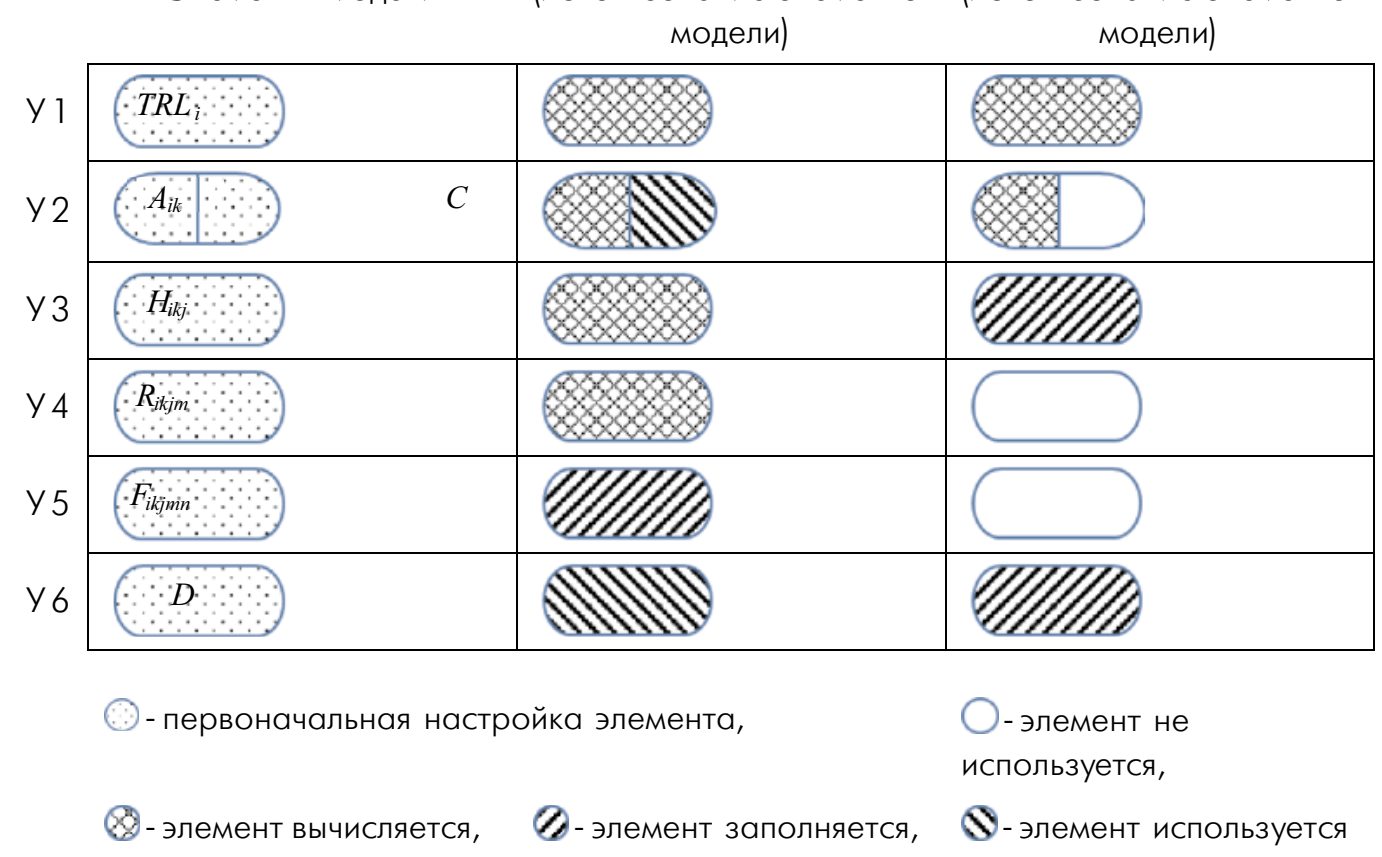

Рис. 1. Структура модели комплексной оценки технологической готовности инновационных научно-технологических проектов 
существующих на рынке решений». Если конкретный показатель выполнен, то его значение принимается равным 1, а если нет, то 0. Для достижимости конкретного уровня технологической готовности проекта, все принадлежащие ему показатели должны иметь значение 1. По степени полноты достигнутых показателей рассчитывается индекс технологической готовности. Маркеры определяют взаимосвязь показателей, построенную на основе анализа требований нормативных документов к выполнению проекта по разработке инновационной продукции (ГОСТ, ОСТ, ТУ и т.п.), например, ни один из показателей 2-го уровня TRL не может быть выполнен ранее, чем достигнут 1-й уровень TRL.
Каждый показатель TRL имеет характеристики (У3), определяющие требования к перечню задач, которые должны быть решены разработчиками для того, чтобы подтвердить выполнение этого показателя, например, для показателя «Проведен анализ существующих на рынке решений» одной из характеристик будет являться «Сформулирован облик технологии/продукта». Каждая характеристика также измеряется в виде значений 0 или 1. Если все характеристики показателя равны 1, то показатель, входящий в конкретный уровень технологической готовности, выполнен, а если хотя бы один из них равен 0, то не выполнен.

Решение каждой из задач для определения характеристики показателя TRL, должно

Таблица 2

\section{Пример построения модели комплексной оценки технологической готовности инновационных научно-технологических проектов на 1-м уровне TRL для ФЦП ИР}

\begin{tabular}{|c|c|c|c|}
\hline Код & & Показатель & Маркер \\
\hline A1.3 & Проведен анализ сущес & твующих на рынке решений & lee A 1.4 \\
\hline Код & \multicolumn{3}{|c|}{ Характеристика показателя } \\
\hline A 1.3 .1 & \multicolumn{3}{|c|}{ Сформулированы ключевые преимущества технологии } \\
\hline A 1.3.2 & \multicolumn{3}{|c|}{ Сформулирован облик технологии/продукта } \\
\hline A 1.3.3 & \multicolumn{3}{|c|}{ Сформулирован набор(ы) ключевых слов для поиска Ru/En } \\
\hline Код & \multicolumn{3}{|c|}{ Фиксируемый результат } \\
\hline A1.3.3.1 & \multicolumn{3}{|l|}{ Ключевые слова (Ru) } \\
\hline Код & Свидетельство & Требования & Документ \\
\hline A1.3.3.1.1 & Ключевые слова (Ru) & \multirow{3}{*}{$\begin{array}{l}\text { 1. Ключевые слова рекомендуется составлять на ос- } \\
\text { нове результата, полезного эффекта и назначения } \\
\text { технологии, для последующего сравнения и оценки } \\
\text { с более широким кругом аналогов объектов оцени- } \\
\text { вания } \\
\text { 2. Ключевые слова и синонимы должны быть прове- } \\
\text { рены по профессиональным словарям, тезаурусам } \\
\text { и классификаторам }\end{array}$} & $\begin{array}{l}\text { Пояснительная } \\
\text { записка / отчет } \\
\text { о поиске }\end{array}$ \\
\hline A 1.3.3.1.2 & $\begin{array}{l}\text { Синонимы ключевых } \\
\text { слов (Ru) }\end{array}$ & & $\begin{array}{l}\text { Пояснительная } \\
\text { записка / отчет } \\
\text { о поиске }\end{array}$ \\
\hline A 1.3.3.1.3 & $\begin{array}{l}\text { Ключевые слова и си- } \\
\text { нонимы (Ru) проверены }\end{array}$ & & $\begin{array}{l}\text { Пояснительная } \\
\text { записка / отчет } \\
\text { о поиске }\end{array}$ \\
\hline A 1.3.3.2 & \multicolumn{3}{|l|}{ Ключевые слова (En) } \\
\hline Код & Свидетельство & Требования & Документ \\
\hline A 1.3.3.2.1 & Ключевые слова (En) & \multirow{3}{*}{$\begin{array}{l}\text { 1. Ключевые слова рекомендуется составлять на осно- } \\
\text { ве результата, полезного эффекта и назначения тех- } \\
\text { нологии, для последующего сравнения и оценки с бо- } \\
\text { лее широким кругом аналогов объектов оценивания } \\
\text { 2. Ключевые слова и синонимы должны быть прове- } \\
\text { рены по профессиональным словарям, тезаурусам } \\
\text { и классификаторам } \\
\text { 3. Ключевые слова (En) не должны быть «точным» пе- } \\
\text { реводом с русского языка, а состоять из слов, яв- } \\
\text { ляющихся ключевыми для соответствующей тематики } \\
\text { (на английском языке) }\end{array}$} & $\begin{array}{l}\text { Пояснительная } \\
\text { записка / отчет } \\
\text { о поиске }\end{array}$ \\
\hline A 1.3.3.2.2 & $\begin{array}{l}\text { Синонимы ключевых } \\
\text { слов (En) }\end{array}$ & & $\begin{array}{l}\text { Пояснительная } \\
\text { записка / отчет } \\
\text { о поиске }\end{array}$ \\
\hline A 1.3.3.2.3 & $\begin{array}{l}\text { Ключевые слова и си- } \\
\text { нонимы (En) проверены }\end{array}$ & & $\begin{array}{l}\text { Пояснительная } \\
\text { записка / отчет } \\
\text { о поиске }\end{array}$ \\
\hline
\end{tabular}


иметь фиксируемый результат (У4), например, одним из результатов выполнения задачи «Сформулированы ключевые слова для поискa Ru/En» будет являться следующий результат - «Ключевые слова (En)».

В свою очередь каждый из результатов, представленных на уровне 4, должен быть подтвержден в виде некоторого набора подтверждающих свидетельств (У5), например, для результата «Ключевые слова (En)» в качестве такого набора подтверждающих свидетельств будет выступать следующий набор: «Ключевые слова (En) - Синонимы ключевых слов (En) Ключевые слова и синонимы (En) проверень».

Каждое из подтверждающих свидетельств должно быть подтверждено, т.е. описание данного свидетельства должно быть представлено разработчиком в каком-либо документе (У6), входящем в состав представляемой по проекту документации, причем к этим документам могут быть предъявлены, в свою очередь, определенные требования, например, отчет о научно-исследовательской работе должен удовлетворять требованиям ГОСТ 7.32-2001.

На рис. 1 также показано, как элементы модели используются при самооценке и экспертной оценке проекта.

Пример структуры модели комплексной оценки технологической готовности инновационных научно-технологических проектов на 1-м уровне TRL, разработанной для ФЦП ИР, представлен в табл. 2, в которой обозначения A1.3 и A1.4 относятся к двум различным показателям TRL.

В представленном в табл. 2 фрагменте модели маркером является утверждение, что показатель Al.3 не может быть выполнен ранее показателя A1.4, т.е. таким образом устанавливается связь между ними на основе очередности их выполнения. Как именно эта связь учитывается в предложенной модели, будет показано ниже.

\section{3.Количественная оценка уровня и индекса технологической готовности проекта}

Оценка уровня технологической готовности проекта осуществляется на основе следующего выражения:

$$
T R L_{i}=i-1+\prod_{k=1}^{K_{i}} A_{i k}
$$

где

$i-$ номер уровня $T R L, i=\overline{1,9}$;

$T R L_{i}$ - численное значение $i$-го уровня TRL;

$A_{i k}$ - численное значение $k$-го показателя, входящего в $i$-й уровень $T R L$

$$
A_{i k}=\left\{\begin{array}{l}
0, \text { если не выполнен } \\
1, \text { если выполнен }
\end{array}\right.
$$

$k$ - номер показателя TRL, входящего в $i$-й уровень TRL, $k=\overline{\left(1, K_{i}\right)}$;

$K_{i}$ - количество входящих в $i$-й уровень TRL показателей.

Оценка значения каждого показателя $A_{i k^{\prime}}$ входящего в выражение (1), осуществляется по его характеристикам с использованием выражения (2):

$$
A_{i k}=\prod_{j=1}^{J} H_{i k j}
$$

где

$j$ - номер характеристики показателя $A_{i k} j=\overline{1, J}$;

$J$ - количество характеристик, описывающих показатель $A_{i k^{\prime}}$

$H_{i k j}$ - численное значение $j$-й характериСтики

$$
H_{i k j}=\left\{\begin{array}{l}
0, \text { если не выполнена } \\
1, \text { если выполнена }
\end{array}\right.
$$

Используя выражения (1) и (2), исполнители проекта могут провести самооценку уровня технологической готовности своего проекта. Действительно, выбирая в качестве ответа на вопрос о достижимости того или иного результата, ответ «ДА» (значение 1) или «НЕТ» (значение 0), можно в итоге провести несложные вычисления и определить численное значение уровня технологической готовности TRL.

В случае же подтверждения достижимости уровня технологической готовности проекта для определения численного значения характеристики $H_{i k j}$ используется другой алгоритм. Расчет значений характеристик показателей производится на основании оценок экспертов в отношении качества представленных 
документов (множества документов $D$ ), подтверждающих наличие тех или иных свидетельств, на основе которых можно вынести суждение о достижении необходимых результатов, входящих в перечень результатов, описывающих характеристику $H_{i k j}$.

Характеристика $H_{i k j}$ может быть представлена в виде множества результатов

$$
H_{i k j}=\left\{R_{i k j m}\right\}, m=\overline{1, M}
$$

(здесь $M$ - количество результатов), каждый из которых, в свою очередь, описывается множеством подтверждающих свидетельств -

$$
R_{i k j m}=\left\{F_{i j k m n}\right\}, n=\overline{1, N}
$$

$(N-$ количество подтверждающих свидетельств $F$ для определенного результата). Свидетельства являются по своей сути элементарными событиями, факт выполнения (или наличия) которых может быть зафиксирован экспертом во время проведения экспертных процедур. Каждое из них не может в полной мере позволить сделать однозначный вывод о достижении определенного результата, это может быть сделано только лишь при наличии всей совокупности подтверждающих свидетельств. В рамках предлагаемой модели каждое свидетельство обладает вероятностной характеристикой $0 \leq p \leq 1$, имеющей следующий смысл: величина $p$ характеризует ту степень уверенности (вероятность достижимости), с которой можно утверждать, что данное свидетельство подтверждает наличие в материалах проектах результата, для описания которого оно создано при условии, что остальные свидетельства, входящие в набор для этого результата, не подтверждены (или отсутствуют). Отметим, что значение этой характеристики $p$ зависит от того, каково качество подтверждающих сведений об этом свидетельстве. Например, наличие определенного документа не гарантирует, что свидетельство будет засчитано экспертом, если содержание документа не удовлетворяет необходимым, заранее определенным, требованиям, как это делается, например, в [10]. Таким образом, эксперт может как повысить, так и понизить значение вероятности $p$. Такой подход к оценке достижимости результатов позволяет варьировать требования к их качеству для различных проектов или проводимых конкурсов по их отбору по следующему правилу: если вероятность достижимости результата по совокупности входящих в него свидетельств будет больше некоторой пороговой

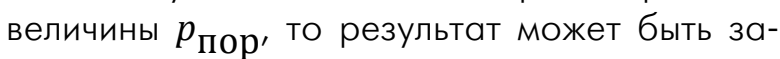
считан, и нет - в противном случае.

Отметим особо, что в предлагаемой модели рекомендуемый набор подтверждающих свидетельств формируется на этапе ее первоначальной настройки, однако, у эксперта есть возможность его расширения.

Итак, для расчета вероятности достижимости результата по совокупности входящих в него подтверждающих свидетельств будем использовать выражение (3):

$$
p\left(R_{i j k m}\right)=1-\prod_{n=1}^{N}\left(1-p\left(F_{i k j m n}\right)\right)
$$

а для расчета величины достигнутого результата выражение (4):

$$
R_{i j k m}=\left\{\begin{array}{l}
1, \text { если } p\left(R_{i j k m}\right) \geq p_{\text {пор }} \\
0, \text { если } p\left(R_{i j k m}\right)<p_{\text {пор }}
\end{array}\right.
$$

Тогда расчет значения величины $H_{i k j}$ при экспертном оценивании проекта может быть выполнен по формуле (5):

$$
H_{i k j}=\prod_{m=1}^{M} R_{i k j m}
$$

Рассмотрим далее, каким образом строится система маркеров для проверки правильности оценивания показателей, входящих в определенный уровень технологической готовности. Поскольку построение этой системы одинаково для каждого уровня TRL, то, без ограничения общности, индекс номера уровня $i$ будем опускать.

Построим матрицу маркеров $\hat{C}=\left\|c_{i j}\right\|, i$, $j=\overline{1, K}$, по следующему правилу:

$$
c_{i j}=\left\{\begin{array}{c}
1 / S_{i}, A_{i} \text { связан с } A_{j} \\
0, \text { в противном случае }
\end{array}\right.
$$

Величина $S_{i}$ равна количеству связей показателя $A_{i}$ (в т.ч. с самим собой) со всеми показателями $A_{k}$. Например, для случая табл. 2, показатель $A_{3}$ связан с показателем $A_{4}$, общее количество связей $S=2$, поэтому, $c_{3,3}=c_{3,4}=0.5$, а остальные значения в строке 3 будут равны 0. Выражение (6) использует 
предположение, что все имеющиеся связи равнозначны, что является достаточным условием для практического использования модели. Очевидно, что если потребуется учесть то обстоятельство, что связи могут обладать весами, выражение (6) может быть модифицировано.

Приведем пример матрицы маркеров, построенной для модели комплексной оценки технологической готовности проектов для 1-го уровня TRL в ФЦП ИР (рис. 2).

Естественно считать, что состояние показателей уровня TRL, определяемых по формуле (1), с использованием выражений $(2)-(5)$, описывается вектором в К-мерном пространстве.

Если вектор рассчитан по результатам проведенной экспертизы, то он должен быть уточнен с использованием выражения (7):

$$
\overrightarrow{\tilde{a}}=\hat{C} \times \vec{a}
$$

Отметим, что матрица построена таким образом, что преобразование единичного вектора также даст единичный вектор, это означает, что если уровень TRL достигнут, то все его показатели остаются равными 1. Полученный вектор, с уточненными значениями показателей уровня TRL может быть использован для определения индекса технологической готовности проекта. Для этого мы должны воспользоваться выражением (8):

ind $(T R L)=T R L-1+\frac{\operatorname{num}\left(\tilde{A}_{k}=1, k=\overline{1, K}\right)}{K}$

В выражении (8) в числителе дроби указано количество показателей уровня $T R L$, значение которых равно 1, а в знаменателе - количество всех показателей, относящихся к определенному уровню технологической готовности. Отметим также, что использование матрицы маркеров позволяет избежать контроля последовательности вопросов анкеты для исполнителей проектов в ходе проведения самооценки уровня технологической готовности, Т.к. в конечном итоге именно с помощью матрицы маркеров по формуле (7) будут рассчитаны окончательные значение показателей уровня TRL.

4. Последовательность определения и подтверждения уровня и индекса технологической готовности проекта

Построенная модель, в соответствии с требованиями к еe разработке, может быть использована как для самооценки технологического уровня проекта, проводимой его исполнителями, так и для его экспертной оценки. Последовательность ее применения может быть в общем случае представлена в виде 2-х этапной процедуры.

Нa 1-м этапе (этапе самооценки):

- исполнитель проекта отвечает на вопросы анкеты в формате («ДА»/«НЕТ»);

- с помощью модели на основании полученных ответов на вопросы анкеты определяется уровень технологической готовности проекта, который рассчитывается по формуле (1) с использованием формулы (2) для определения значений показателей TRL. При этом выбор ответа «ДА»на вопрос анкеты в отношении какого-либо показателя означает,

$$
\hat{C}=\left\|\begin{array}{|cccccccccc}
1 & 0 & 0 & 0 & 0 & 0 & 0 & 0 & 0 & 0 \\
1 / 2 & 1 / 2 & 0 & 0 & 0 & 0 & 0 & 0 & 0 & 0 \\
0 & 0 & 1 & 0 & 0 & 0 & 0 & 0 & 0 & 0 \\
0 & 1 / 4 & 1 / 4 & 1 / 4 & 0 & 0 & 0 & 1 / 4 & 0 & 0 \\
0 & 0 & 0 & 0 & 1 / 2 & 1 / 2 & 0 & 0 & 0 & 0 \\
0 & 1 / 4 & 1 / 4 & 1 / 4 & 0 & 1 / 4 & 0 & 0 & 0 & 0 \\
0 & 0 & 1 / 2 & 0 & 0 & 0 & 1 / 2 & 0 & 0 & 0 \\
0 & 0 & 1 / 3 & 0 & 0 & 0 & 1 / 3 & 1 / 3 & 0 & 0 \\
0 & 0 & 0 & 0 & 0 & 1 / 2 & 0 & 0 & 1 / 2 & 0 \\
0 & 0 & 0 & 0 & 0 & 0 & 1 / 2 & 0 & 0 & 1 / 2
\end{array}\right\|
$$

Рис. 2. Матрица маркеров для модели комплексной оценки технологической готовности проектов для 1-го уровня TRL 
что ему в модели присваивается значение 1, в противном случае - значение 0;

- если уровень технологической готовности проекта не подтвержден, осуществляется переход на предыдущий уровень;

- самооценка может продолжаться до тех пор, пока с помощью модели не будет подтвержден текущий (с точки зрения проводимой самооценки) уровень технологической готовности проекта;

- в случае подтверждения технологического уровня проекта исполнителям рекомендуется перечень документов, которые должны быть представлены для его подтверждения независимыми экспертами.

На 2-м этапе (этапе экспертной оценки):

- эксперт оценивает качество и полноту подтверждающих фактов/документов и определяет их вероятностные характеристики;

- по модели рассчитываются значения вероятностей достижимости результатов, характеристик и показателей TRL по формуле (7);

- с помощью матрицы маркеров рассчитанные значения показателей TRL корректируются;

- с помощью модели рассчитывается уровень технологической готовности проекта по формуле (7) и индекс технологической готовности проекта по формуле (8).

Если же мы говорим об экспертной оценке, то значение показателей TRL рассчитывается с использованием выражения (7), после чего определяется уровень технологической готовности по формуле (1) и индекс технологической готовности по формуле (8).

\section{ЗАКЛЮЧЕНИЕ}

В статье описана модель комплексной оценки технологической готовности инновационных научно-технологических проектов, разработанная в рамках методологии TPRL, однако в то же время расширяющая границы ее использования. В частности, данная модель предлагает единые метрики оценки проектов не только в ходе их выполнения, но и на этапе проведения конкурсного отбора проектов.
Разработка модели проведена авторским коллективом на основе требований, которые были сформулированы по итогам апробации методологии TPRL на практике.

Модель обладает как свойством универсальности, т.е. ее структура и математическая формализация не связаны с конкретными техническими дисциплинами и разделами науки, а, следовательно, могут быть использованы различными институтами поддержки, так и свойством комплексности - оценка проектов проводится не только по шкале технологической готовности, но и характеристикам других шкал готовности, среди которых:

- Производственная готовность (MRL);

- Инженерная готовность (ERL);

- Организационная готовность (ORL);

- Преимущества и риски (BRL);

- Рыночная готовность и коммерциализация (CRL).

Применение модели на практике позволяет учитывать вариативность предъявляемых требований к получаемым результатам проекта за счет использования вероятностных характеристик, связанных с оценкой качества и полноты этих результатов.

Количественные оценки уровня технологической готовности проекта, полученные с помощью модели, могут быть использованы для принятия различных управленческих решений, например, для разработки плана-графика работ, плана финансирования, в т ч. определения соотношения долей бюджетного и внебюджетного финансирования в рамках программ, реализуемых различными институтами поддержки, а также других решений.

Модель может быть применена для формализации и оптимизации процедур экспертной оценки проектов на основе унифицированных подходов и требований к формированию системы критериев для экспертной оценки, что имеет решающее значение для комплексных научно-технических проектов, жизненный цикл которых реализуется различными институтами поддержки. 


\section{ЛИТЕРАТУРА}

1. Mankins J.C. (1995) Technology readiness levels / Advanced Concepts Office of Space Access and 1995. https://www.colorado.edu/ASEN/ asen3036/TECHNOLOGYREADINESSLEVELS.pdf.

2. Sadin S.R., Povinelli F.P., Rosen R. (1989) The NASA technology push towards future space mission systems // Acta Astronautica. V. 20. P. 73-33.

3. Hirshorn S., Sharon J. (2016) Final Report of the NASA Technology Readiness Assessment (TRA) Study Team. NASA. $63 \mathrm{p}$.

4. ISO 16290:2013 (2013) Space systems - Definition of the Technology Readiness Levels (TRLs) and their criteria of assessment.

5. ГОСТ Р 56861-2016 (2016) Система управления жизненным циклом. Разработка концепции изделия и технологий. Общие положения. Москва. 11 с.

6. ГОСТ Р 57194.1-2016 (2016) Трансфер технологий. Общие положения. Москва. 9 с.

7. GAO TRA Guide: Best Practices for Evaluating the Readiness of Technology (2016) GAO-16-410G. $147 \mathrm{p}$.

8. Петров А.Н., Сартори А.В., Филимонов А.В. (2016) Комплексная оценка состояния научно-технических проектов через уровень готовности технологий // Экономика науки. Т. 2. № 4. C. $244-260$.

9. Тихомиров И.А., Жебель В.В., Каменская М.А., Комаров А.В. (2017) Оценка качества патентных исследований // Интеллектуальная собственность. Промышленная собственность. № 5. C. $47-54$.
10. Комаров А.В., Тихомиров И.А., Жебель В.В. (2017) Информационно-аналитический сервис для обеспечения экспертной оценки качества патентных исследований // Научный сервис в сети Интернет: труды XIX Всероссийской научной конференции. М.: ИПМ им. М.В. Келдыша. C. 281-289.

11. TRL Worksheet (2017) NASA ESTO. https://esto. nasa.gov/files/TRL_Worksheet_11-30-10.xls.

12. TRL/CRL Calculator (2018) NYSERDA Portal. https://portal.nyserda.ny.gov/servlet/servlet.FileD ownload? file=00P+0000004FyRNEA0.

13. Дмитренко И.П., Криворученко В.С. (2015) Калькулятор готовности технологий (TR) // Материалы IV Международной научно-практической конференции «Общество, наука, инновации». Москва.

14. William L.N., Brian C.K., Roger J., Dziegiel Jr. (2003) Air Force Research Laboratory (AFRL). Technology Readiness Calculator // NDIA system engineering conference.

15. Сартори A. (2016) Инновационные проекты и их внедрение: Методология определения степени готовности инновационных проектов TPRL. https://acc.xpir.ru/assets/files/\%D0\%9C\% D0\%B5\%D 1\%82\%D0\%BE\%D0\%B4\%D0\%BE\%D 0\%BB\%D0\%BE\%D0\%B3\%D0\%B8\%D 1\%8F\%20 TPRL.pdf.

16. Перспективы российских ИТ-разработок на глобальном рынке (2017) Руссофт. http://www. russoft.ru/files/ITexport.pdf.

\section{REFERENCES}

1. Mankins J.C. (1995) Technology readiness levels / Advanced Concepts Office of Space Access and 1995. https://www.colorado.edu/ ASEN/asen3036/TECHNOLOGYREADINESSLEVELS.pdf.

2. Sadin S.R., Povinelli F.P., Rosen R. (1989) The NASA technology push towards future space mission systems // Acta Astronautica. V. 20. P. 73-33.

3. Hirshorn S., Sharon J. (2016) Final Report of the NASA Technology Readiness Assessment (TRA) Study Team. NASA. 63 p.

4. ISO 16290:2013 (2013) Space systems - Definition of the Technology Readiness Levels (TRLs) and their criteria of assessment.

5. GOST P 56861-2016 (2016) Lifecycle management system. Development of concepts and technologies. General provisions. Moscow. 11 p.

6. GOST P 57194.1-2016 (2016) Technology transfer. General provisions. Moscow. 9 p.
7. GAO TRA Guide: Best Practices for Evaluating the Readiness of Technology (2016) GAO-16-410G. $147 \mathrm{p}$.

8. Petrov A.N., Sartory A.V., Filimonov A.V. (2016) Comprehensive assessment of the status scientific and technical projects using Technology Project Readiness Level // The Economics of Science. V. 2. № 4. P. 244-260.

9. Tihomirov I.A., Zhebel' V.V., Kamenskaja M.A., Komarov A.V. (2017) Evaluation of the quality of patent research // Intellectual Property. Industrial property. № 5. P. 47-54.

10. Komarov A.V., Tihomirov I.A., Zhebel' V.V. (2017) Informational and analytical service for providing expert evaluation of the quality of patent research // Scientific service in the Internet: Proceedings of the XIX All-Russian Scientific Conference. Moscow: Keldysh Institute of Applied Mathematics. P. 281-289. 
11. TRL Worksheet (2017) NASA ESTO. https://esto. nasa.gov/files/TRL_Worksheet_11-30-10.xls.

12. TRL/CRL Calculator (2018) NYSERDA Portal. https://portal.nyserda.ny.gov/servlet/servlet.FileDo wnload? file $=00$ Pt0000004FyRNEA0.

13. Dmitrenko I.P., Krivoruchenko V.S. (2015) Calculator of technology readiness (TR) // Proceedings of the IV International Scientific and Practical Conference: Society, Science, Innovations. Moscow.

14. William L.N., Brian C.K., Roger J., Dziegiel Jr. (2003) Air Force Research Laboratory (AFRL). Technology
Readiness Calculator // NDIA system engineering conference.

15. Sartory A. (2016) Innovative projects and their implementation: Methodology for determining the degree of readiness of innovation projects TPRL. https://acc.xpir.ru/assets/files/\%D0\%9C\%D0\%B5 $\% \mathrm{D} 1 \% 82 \% \mathrm{D} 0 \% \mathrm{BE} \% \mathrm{D} 0 \% \mathrm{~B} 4 \% \mathrm{D} 0 \% \mathrm{BE} \% \mathrm{D} 0 \% \mathrm{BB} \% \mathrm{D}$ 0\%BE\%D0\%B3\%D0\%B8\%D1\%8F\%20TPRL.pdf.

16. Prospects of Russian IT developments in the global market (2017) Russoft. http://www.russoft.ru/files/ ITexport.pdf.

UDC 338.28, 378.4

Komarov A.V., Petrov A.N., Sartory A.V. The model of integrated assessment of technological readiness

of innovative scientific and technological projects (Directorate of State Scientific and Technical Programmes,

Presnensky Val Street, 19, building 1, Moscow, Russia, 123557)

I Abstract. The article describes the model of integrated assessment of technological readiness of innovative scientific I and technological projects, which is an integral part of the methodology of expert evaluation of projects. The

I model can be used both at the stage of selection of applications for project financing, and for creating tools for I measuring the level of technological readiness of projects in the course of their implementation. The application of

I the proposed model will make it possible to formulate unified approaches and requirements to the formation of a

I system of criteria for the expert evaluation of innovative scientific and technological projects at various stages of

I their life cycle that can be used by various development institutions, and for project implementers - to determine the

I degree of readiness of the results obtained for industrial implementation.

I Keywords: scientific and technological project, level of technology readiness, technological readiness index, TPRL,

IRL, UGT, innovation, analysis, model.

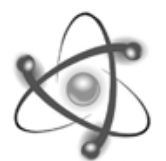

B рамках реализации Стратегии научно-технологического развития Российской Федерации Минобрнауки России объявлен сбор предложений по созданию проектов класса «мегасайенс».

Предложения должны быть нацелены на создание и развитие сети уникальных научных установок класса «мегасайенс» на территории Российской Федерации и участия Российской Федерации в зарубежных проектах класса «мегасайенс». Под уникальной научной установкой класса мегасайенс понимается не имеющая аналогов в мире физическая (комплекс научного оборудования) или цифровая (информационная) инфраструктура, в том числе распределенного типа, функционирующая как единое целое, и ориентированная на получение научных результатов, достижение которых невозможно на других установках мира. При этом финансирование создания и эксплуатации такой установки должно осуществляться на основе международного научно-технического сотрудничества.

Правительственной комиссией по высоким технологиям и инновациям ранее уже получили одобрение на реализацию на территории Российской Федерации 6 проектов класса мегасайенс: Комплекс сверхпроводящих колец на встречных пучках тяжёлых ионов NICA («Комплекс NICA»); Международный центр нейтронных исследований на базе высокопоточного исследовательского реактора ПИК (МЦНИ ПИК); Токамак с сильным магнитным полем (Игнитор); Ускорительный комплекс со встречными электрон-позитронными пучками (Супер Чарм-Тау фабрика); Международный центр исследований экстремальных световых полей (ЦИЭС); Рентгеновский источник синхротронного излучения четвертого поколения (ИССИ-4).

Предложения по созданию проектов класса «мегасайенс» на территории России и присоединения к проектам, реализуемым за рубежом, могут подаваться как от организации, так и от коллектива ученых и должны направляться в электронном виде на адрес электронной почты: mega@mon.gov.ru до 26 апреля 2018 г.

Контактное лицо: Мастерских Евгений Сергеевич, тел.: (495) 530-68-44, mega@mon.gov.ru.

Источник: https://xpir.ru/news 